\title{
Pharmacokinetic Concentration Numeric Result in Standard Unit
}

National Cancer Institute

\section{Source}

National Cancer Institute. Pharmacokinetic Concentration Numeric Result in Standard

Unit. NCl Thesaurus. Code C87965.

The numerical identifier of a pharmacokinetic concentration result in standard units. 